The Nepal Math. Sc. Report

Vol. 34, No.1 and 2, 2016

\title{
INVARIANCE OF DOMAIN FOR OPERATORS OF CLASS $\mathcal{A}_{G}\left(S_{+}\right)$
}

\author{
DHRUBA R. ADHIKARI \\ Department of Mathematics \\ Kennesaw State University, Gerogia 30060, USA \\ dadhikar@kennesaw.edu
}

\begin{abstract}
Let $X$ be a real reflexive Banach space and $X^{*}$ its dual space. Let $T: X \supset D(T) \rightarrow 2^{X^{*}}$ an operator of class $\mathcal{A}_{G}\left(S_{+}\right)$, where $G \subset X$ is open. An invariance of domain result for $T$ is established. This result extends a similar result of Park for single-valued operators of type $\left(S_{+}\right)$. The Skrypnik's topological degree theory is used, utilizing approximating schemes of mappings of class $\mathcal{A}_{G}\left(S_{+}\right)$, along with the methodology of a recent invariance of domain result by Kartsatos and the author.
\end{abstract}

Key Words: Browder and Skrypnik degree theory, invariance of domain, bounded demicontinuous operator of type $\left(S_{+}\right)$

AMS (MOS) Subject Classification. Primary 47H14; Secondary 47H05, 47H11

\section{InTRODUCTION-PRELIMINARIES}

Let $X$ be a real reflexive Banach space with $X^{*}$ its dual space. The norms of $X, X^{*}$ will be denoted by $\|\cdot\|$ and will be understood from the context in which the symbol is used. We denote by $\left\langle x^{*}, x\right\rangle$ the value of the functional $x^{*} \in X^{*}$ at $x \in X$. The symbols $\partial D, \bar{D}$ denote the strong boundary and closure of the set $D$, respectively. The symbol $B\left(x_{0}, r\right)$ denotes the open ball of radius $r$ with center at $x_{0}$.

If $\left\{x_{n}\right\}$ is a sequence in $X$, we denote its strong convergence to $x_{0}$ in $X$ by $x_{n} \rightarrow x_{0}$ and its weak convergence to $x_{0}$ in $X$ by $x_{n} \rightarrow x_{0}$. An operator $T: X \supset D(T) \rightarrow Y$ is said to be "bounded" if it maps bounded subsets of the domain $D(T)$ onto bounded subsets of $Y$. The operator $T$ is said to be "compact" if it maps bounded subsets of $D(T)$ onto relatively compact subsets in $Y$. It is said to be "demicontinuous" if it is strong-weak continuous on $D(T)$. The symbols $\mathbf{R}$ and $\mathbf{R}_{+}$denote $(-\infty, \infty)$ and $[0, \infty)$, respectively. The normalized duality mapping $J: X \supset D(J) \rightarrow 2^{X^{*}}$ is defined by

$$
J x=\left\{x^{*} \in X^{*}:\left\langle x^{*}, x\right\rangle=\|x\|^{2},\left\|x^{*}\right\|=\|x\|\right\}, x \in X .
$$

The Hahn-Banach theorem ensures that $D(J)=X$, and therefore $J: X \rightarrow 2^{X^{*}}$ is a multivalued mapping defined on the whole space $X$. By a well-known renorming theorem due to Trojanski [15], one can always renorm the reflexive Banach space $X$ with an equivalent norm with respect to which both $X$ and $X^{*}$ become locally uniformly convex (therefore strictly

Received May 13, 2016 
convex). Henceforth, we assume that $X$ is a locally uniformly convex reflexive Banach space. With this setting, the normalized duality mapping $J$ is single-valued homeomorphism from $X$ onto $X^{*}$.

For a multivalued operator $T$ from $X$ to $X^{*}$, we write $T: X \supset D(T) \rightarrow 2^{X^{*}}$, where $D(T)=\{x \in X: T x \neq \emptyset\}$ is the effective domain of $T$. We denote by $G r(T)$ the graph of $T$, i.e., $\operatorname{Gr}(T)=\{(x, y): x \in D(T), y \in T x\}$.

An operator $T: X \supset D(T) \rightarrow 2^{X^{*}}$ is said to be "monotone" if for every $x, y \in D(T)$ and every $u \in T x, v \in T y$ we have

$$
\langle u-v, x-y\rangle \geq 0
$$

A monotone operator $T$ is said to be "maximal monotone" if $\operatorname{Gr}(T)$ is maximal in $X \times X^{*}$, when $X \times X^{*}$ is partially ordered by the set inclusion. In our setting, a monotone operator $T$ is maximal if and only if $R(T+\lambda J)=X^{*}$ for all $\lambda \in(0, \infty)$.

Definition 1.1. An operator $C: X \supset D(C) \rightarrow X^{*}$ is said to be of type $\left(S_{+}\right)$if for every sequence $\left\{x_{n}\right\} \subset D(C)$ with $x_{n} \rightarrow x_{0}$ in $X$ and

$$
\limsup _{n \rightarrow \infty}\left\langle C x_{n}, x_{n}-x_{0}\right\rangle \leq 0,
$$

we have $x_{n} \rightarrow x_{0} \in \overline{D(C)}$ in $X$.

Definition 1.2. The family $C(t): X \supset D \rightarrow X^{*}, t \in[0,1]$, of operators is said to be a "homotopy of type $\left(S_{+}\right)$" if for any sequences $\left\{x_{n}\right\} \subset D$ with $x_{n} \rightarrow x_{0}$ in $X$ and $\left\{t_{n}\right\} \subset[0,1]$ with $t_{n} \rightarrow t_{0}$ and

$$
\limsup _{n \rightarrow \infty}\left\langle C\left(t_{n}\right) x_{n}, x_{n}-x_{0}\right\rangle \leq 0
$$

we have $x_{n} \rightarrow x_{0}$ in $X, x_{0} \in D$ and $C\left(t_{n}\right) x_{n} \rightarrow C\left(t_{0}\right) x_{0}$ in $X^{*}$. A homotopy of type $\left(S_{+}\right)$ is "bounded" if the set

$$
\{C(t) x \mid t \in[0,1], x \in D\}
$$

is bounded.

We now define the class $\mathcal{A}_{G}\left(S_{+}\right)$of multivalued operators, where is introduced by Kittila in [8].

Definition 1.3. Let $G$ be an open subset of $X$. An operator $T: X \supset D(T) \rightarrow 2^{X^{*}}$ is of class $\mathcal{A}_{G}\left(S_{+}\right)$if there exists a sequence $\left(T_{n}\right)$ (called an approximating sequence of $T$ ) of bounded demicontinuous mappings of type $\left(S_{+}\right)$from $\bar{G}$ to $X^{*}$ with the following properties.

(A1) For each $C>0$ there exists $K \geq 0$ such that $\left\langle T_{n}(x), x\right\rangle \geq-K$ for all $u \in \bar{G},\|x\| \leq C$ and for all $n \in \mathbb{N}$.

(A2) Let $\left\{t_{n}\right\} \subset[0,1],\left(u_{n}\right) \subset \bar{G}$ and let $\left\{T_{m_{n}}\right\}$ be any subsequence of $\left\{T_{n}\right\}$. If $t_{n} \rightarrow 0$, $x_{n} \rightarrow x$ in $X$ and $t_{n} T_{m_{n}}\left(x_{n}\right) \rightarrow z$ in $X^{*}$, then $z=0$.

(A3) Let $\left\{x_{n}\right\} \subset \bar{G}$ and $\left\{T_{m_{n}}\right\}$ be any subsequence of $\left\{T_{n}\right\}$. If $x_{n} \rightarrow x$ in $X, T_{m_{n}}\left(x_{n}\right) \rightarrow w$ in $X^{*}$ and

$$
\left.\limsup _{n \rightarrow \infty}\left\langle T_{m_{n}} x_{n}\right), x_{n}\right\rangle \leq\langle w, x\rangle
$$

then $x_{n} \rightarrow x$ in $X, x \in D(T)$ and $w \in T(x)$. 
The main purpose of this paper is to give an invariance of domain result for operators of class $\mathcal{A}_{G}\left(S_{+}\right)$. These operators are generalizations of operators of type $\left(S_{+}\right)$and have an approximating scheme in terms of bounded demicontinuous $\left(S_{+}\right)$-operators as given in Definition 1.3, and therefore this paper generalizes results of Park in [10]. A multivalued degree for operators in $\mathcal{A}_{G}\left(S_{+}\right)$is developed by Kittila in [8] via the Skyrpnik's degrees (cf. [14]). The methodologies of [8] and recent papers of the author and Kartsatos [1] and Kartsatos and Skrypnik [7] along with properties of the Skrypnik's degree in [14] have been utilized.

Invariance of domain results date as far back as Brouwer [3] for continuous injection in $\mathbf{R}^{n}$. Schauder [13] extended the Brouwer's invariance of domain result to infinite dimensional Banach spaces for compact displacements of the identity, i.e. for operators of the form $I+C$ with $C$ compact. Tromba [16] extended the Schauder's result to Fredholm maps of index zero. For other results on invariance of domain under continuity or demicontinuity assumptions on the main operators, the reader is referred to Berkovits [2], Deimling [4], Kartsatos [5], Nagumo [9], Petryshyn [11, 12] (for $A$-proper mappings), Skrypnik [14, p.59] and the references therein. For the existence of pathwise connected set in the ranges of certain operators, the reader is referred to [6] and the references therein.

\section{Invariance of Domain Result for Operators of Class $\mathcal{A}_{G}\left(S_{+}\right)$}

In this section, we first prove a result that has to do with placing a pathwise connected set in the range of the operators of class $\mathcal{A}_{G}\left(S_{+}\right)$. As a consequence of this result, we then obtain an invariance of domain result for operators in class $\mathcal{A}_{G}\left(S_{+}\right)$. It is well-known (cf. Kittila [8, p.13]) that a densely defined maximal monotone operator $A: X \supset D(T) \rightarrow 2^{X^{*}}$, $0 \in D(A), 0 \in A(0)$ satisfies $A+T$ is in $\mathcal{A}_{G}\left(S_{+}\right)$whenever $T \in \mathcal{A}_{G}\left(S_{+}\right)$. In particular, $T+J \in \mathcal{A}_{G}\left(S_{+}\right)$.

Proposition 2.1. Let $T: X \supset D(T) \rightarrow 2^{X^{*}}$ be of class $\mathcal{A}_{G}\left(S_{+}\right)$with an approximating sequence $T_{n}$, where $G \subset X$ is open and bounded. Assume that $T+\epsilon J\left(\cdot-x_{0}\right)$ is injective on $G$ for each $\epsilon \geq 0$ and for every $x_{0} \in D(T)$. Moreover, assume that for each $x_{0} \in D(T)$ and for each $r>0$, there exists a bounded $\phi: \mathbf{R}^{+} \rightarrow \mathbf{R}^{+}$such that $\left\langle T_{n} x, x_{0}\right\rangle \leq \phi(\|x\|)$ for all $x \in \bar{G} \cap \partial B(0, r)$ and for all large $n$. For a pathwise connected set $M \subset X^{*}$, assume that $T(D(T) \cap G) \cap M \neq \emptyset$ and $T(D(T) \cap \partial G) \cap M=\emptyset$. Then $M \subset T(D(T) \cap G)$.

Proof: Let $y_{0} \in T(D(T) \cap G) \cap M$. Then there exists $x_{0} \in D(T) \cap G$ such that $y_{0} \in T\left(x_{0}\right)$. Let $p \in M$. Take $f:[0,1] \rightarrow M$ be a path in $M$ such that $f(0)=y_{0}$ and $f(1)=p$. We now claim that there exist $n_{0} \in \mathbb{N}$ such that

$$
T_{n} x+\frac{1}{n} J\left(x-x_{0}\right)=f(t)
$$

has no solution $x \in \partial G$ for any $t \in[0,1]$ and for all $n \geq n_{0}$. Assuming on the contrary and without loosing the generality, let $\left\{x_{n}\right\} \subset \partial G$ with $x_{n} \rightarrow x$ and $\left\{t_{n}\right\} \subset[0,1]$ with $t_{n} \rightarrow t_{0}$ be such that

$$
T_{n} x_{n}+\frac{1}{n} J\left(x_{n}-x_{0}\right)=f\left(t_{n}\right)
$$


This implies that $T_{n} x_{n} \rightarrow f\left(t_{0}\right)$. Since $x_{n} \rightarrow x$, we have

$$
\limsup _{n \rightarrow \infty}\left\langle T_{n} x_{n}, x_{n}\right\rangle \leq\left\langle f\left(t_{0}\right), x\right\rangle
$$

and then by the condition $\left(A_{3}\right)$ of Definition 1.1, we have $x_{n} \rightarrow x, x \in D(T)$ and $f\left(t_{0}\right) \in T x$. Since $f\left(t_{0}\right) \in M$ and $x \in D(T) \cap \partial G$, we have a contradiction to $T(D(T) \cap \partial G) \cap M=\emptyset$.

We now consider the following homotopy equation:

$$
H_{n}(x, t) \equiv T_{n} x+\frac{1}{n} J\left(x-x_{0}\right)-f(t)=0 .
$$

We have already established that this equation has no solution on $\partial G$ for sufficiently large $n$ and for any $t \in[0,1]$, and therefore this is an admissible homotopy of type $\left(S_{+}\right)$.

We next consider the homotopy equation

$$
G_{n}(x, t) \equiv(1-t)\left(T_{n} x+\frac{1}{n} J\left(x-x_{0}\right)-y_{0}\right)+t J\left(x-x_{0}\right)=0 .
$$

We show that (2.3) has no solution on $\partial G$ for any $t \in[0,1]$ and for all $n \geq n_{0}$. If not, let $\left\{x_{n}\right\} \subset \partial B\left(x_{0}, r\right)$ with $x_{n} \rightarrow x$ and $\left\{t_{n}\right\} \subset[0,1]$ with $t_{n} \rightarrow t_{0}$ such that

$$
\left(1-t_{n}\right)\left(T_{n} x_{n}+\frac{1}{n} J\left(x_{n}-x_{0}\right)-y_{0}\right)+t_{n} J\left(x_{n}-x_{0}\right)=0 .
$$

Since (2.1) has no solution on $\partial G$ for any $n \geq n_{0}$ and $t \in[0,1]$, we have that $t_{n}=0$ is impossible for all large $n$. Since $J$ is injective, $t_{n}=1$ is also imposssible. Suppose $t_{0}=1$. The equation (2.4) implies

$$
\left.\left(1-t_{n}\right)\left\langle T_{n} x_{n}, x_{n}-x_{0}\right\rangle+a_{n}\left\|x_{n}-x_{0}\right\|^{2}-\left(1-t_{n}\right)\left\langle y_{0}, x_{n}-x_{0}\right\rangle\right)=0,
$$

where

$$
a_{n}=\frac{1-t_{n}}{n}+t_{n}
$$

We may assume that $x_{n} \rightarrow x$ and let $C>0$ be such that $\left\|x_{n}\right\| \leq C$ for all $n$. By the condition $\left(A_{1}\right)$, there exists a $K>0$ such that $\left\langle T_{n} x_{n}, x_{n}\right\rangle \geq-K$ for all $n$. Also, by the hypothesis, there exists a bounded function $\phi: \mathbf{R}_{+} \rightarrow \mathbf{R}_{+}$such that $\left\langle T_{n} x_{n}, x_{0}\right\rangle \leq \phi\left(\left\|x_{n}\right\|\right)$ for all $n$. Then from (2.4), we have

$$
-\left(1-t_{n}\right) K-\left(1-t_{n}\right) \phi\left(\left\|x_{n}\right\|\right)+a_{n}\left\|x_{n}-x_{0}\right\|^{2}-\left(1-t_{n}\right)\left\langle y_{0}, x_{n}-x_{0}\right\rangle \leq 0 .
$$

Since $t_{n} \rightarrow 1, a_{n} \rightarrow 1$ and $\phi$ is bounded, letting $n \rightarrow \infty$ in (2.6), we obtain $x_{n} \rightarrow x_{0} \in \partial G$, which is a contradiction.

Next, we assume that $t_{0} \in[0,1)$. If $t_{0}=0$, define $\alpha_{n}=\frac{t_{n}}{1-t_{n}}$. Then $\alpha_{n} \downarrow 0$ and

$$
T_{n} x_{n}+\left(\frac{1}{n}+\alpha_{n}\right) J\left(x_{n}-x_{0}\right)=y_{0} .
$$

This equation is like (2.1) for which we have already established the impossibility of solutions on $\partial G$ with $f(t) \equiv y_{0}$. For the remaining case, $t_{0} \in(0,1)$, we define

$$
\beta_{n}=\frac{1}{n}+\frac{t_{n}}{1-t_{n}}
$$

Then $\beta_{n} \rightarrow \beta_{0}:=\frac{t_{0}}{1-t_{0}}>0$. Then the equation becomes

$$
T_{n} x_{n}+\beta_{n} J\left(x_{n}-x_{0}\right)=y_{0} .
$$


If

$$
\limsup _{n \rightarrow \infty}\left\langle T_{n} x_{n}, x_{n}-x\right\rangle>0,
$$

then, by passing to a subsequence, let

$$
q:=\lim _{n \rightarrow \infty}\left\langle T_{n} x_{n}, x_{n}-x\right\rangle>0 .
$$

In view of $(2.7)$, this yields

$$
\limsup _{n \rightarrow \infty}\left\langle\beta_{n} J\left(x_{n}-x_{0}\right),\left(x_{n}-x_{0}\right)-\left(x-x_{0}\right)\right\rangle=-q<0 .
$$

Since $\beta_{n} \rightarrow \beta_{0}>0$ and $J$ is of type $\left(S_{+}\right)$, we obtain $x_{n} \rightarrow x \in \partial G$. From this and (2.7), we get $T_{n} x_{n} \rightarrow w:=-\beta_{0} J\left(x-x_{0}\right)+y_{0}$. By the condition $\left(A_{3}\right)$, we obtain $x \in D(T)$ and $w \in T(x)$, i.e. $y_{0} \in T(x)+\beta_{0} J\left(x-x_{0}\right)$. This leads to a contradiction to the injectivity of $T+\epsilon J\left(\cdot-x_{0}\right)$ because $x \neq x_{0}$.

Thus, $H_{n}(x, t)$ and $G_{n}(x, t)$ are admissible homotopies for the Skrypnik's degree, $d_{\left(S_{+}\right)}$, for the mappings of type $\left(S_{+}\right)$. By the invariance of the degree under these homotopies, we obtain

$$
\begin{aligned}
d_{\left(S_{+}\right)}\left(T_{n}+\frac{1}{n} J\left(\cdot-x_{0}\right)-p, G, 0\right) & =d_{\left(S_{+}\right)}\left(H_{n}(\cdot, 1), G, 0\right) \\
& =d_{\left(S_{+}\right)}\left(H_{n}(\cdot, 0), G, 0\right) \\
& =d_{\left(S_{+}\right)}\left(G_{n}(\cdot, 1), G, 0\right) \\
& =d_{\left(S_{+}\right)}\left(G_{n}(\cdot, 0), G, 0\right) \\
& =d_{\left(S_{+}\right)}\left(J\left(\cdot-x_{0}\right), G, 0\right)=1 .
\end{aligned}
$$

Here, the last equality follows by considering the $\left(S_{+}\right)$-homotopy

$$
Q(x, t)=(1-t) J\left(x-x_{0}\right)+t J x
$$

with a continuous curve $y(t)=t J x_{0}$ so that

$$
\begin{aligned}
d_{\left(S_{+}\right)}\left(J\left(\cdot-x_{0}\right), G, 0\right) & =d_{\left(S_{+}\right)}(Q(\cdot, 0), G, 0) \\
& =d_{\left(S_{+}\right)}\left(Q(\cdot, 1), G, J x_{0}\right) \\
& =d_{\left(S_{+}\right)}\left(J, G, J x_{0}\right)=1 .
\end{aligned}
$$

Therefore, for every $n$, there is $x_{n} \in G$ such that

$$
H_{n}\left(x_{n}, 1\right)=0
$$

i.e.

$$
T_{n} x_{n}+\frac{1}{n} J\left(x_{n}-x_{0}\right)=p,
$$

which implies $T_{n} x_{n} \rightarrow p$. By the condition $\left(A_{3}\right)$, we deduce that $x_{n} \rightarrow x \in \bar{G}, x \in D(T)$, and $p \in T(x)$. Since $T(D(T) \cap \partial G) \cap M=\emptyset$, we can only have $x \in G$. Since $p$ was an arbitrary point in $M$, we obtain $M \subset T(D(T) \cap G)$.

We now apply Proposition 2.1 to obtain the following invariance of domain result. 
Theorem 2.2 (Invariance of Domain). Let $T: X \supset D(T) \rightarrow 2^{X^{*}}$ be of class $\mathcal{A}_{G}\left(S_{+}\right)$ with an approximating sequence $T_{n}$, where $G \subset X$ is open. Assume that $T+\epsilon J\left(\cdot-x_{0}\right)$ is locally injective on $G$ for each $\epsilon \geq 0$ and for every $x_{0} \in D(T)$. Moreover, assume that for each $x_{0} \in D(T)$ and for each $r>0$, there exists a bounded $\phi: \mathbf{R}^{+} \rightarrow \mathbf{R}^{+}$such that $\left\langle T_{n} x, x_{0}\right\rangle \leq \phi(\|x\|)$ for all $x \in \bar{G} \cap \partial B(0, r)$ and for all large $n$. Then $T(D(T) \cap G)$ is open.

Proof: Let $y_{0} \in T(D(T) \cap G)$. Then there exists $x_{0} \in G$ such that $y_{0} \in T\left(x_{0}\right)$. Since $T$ is locally injective on $G$, there is $r>0$ such that $T$ is injective on $\overline{B\left(x_{0}, r\right)} \cap D(T)$, where $\overline{B\left(x_{0}, r\right)} \subset G$. It is then clear that $y_{0} \notin T\left(D(T) \cap \partial B\left(x_{0}, r\right)\right)$. By Lemma 3.2 in [8], there exits $n_{0} \in \mathbb{N}$ such that $y_{0} \notin T_{n}\left(\partial B\left(x_{0}, r\right)\right)$ for all $n \geq n_{0}$, where $\left\{T_{n}\right\}$ is an approximating sequence of $T$ as in the hypothesis of the theorem.

We claim that there exists $\delta>0$ such that $B\left(y_{0}, \delta\right) \cap T\left(D(T) \cap \partial B\left(x_{0}, r\right)\right)=\emptyset$. Assume the contrary, and let $w_{n} \in B\left(y_{0}, 1 / n\right) \cap T\left(D(T) \cap \partial B\left(x_{0}, r\right)\right)$. Then $y_{n} \rightarrow y_{0}$ and $w_{n} \in T\left(x_{n}\right)$ with $x_{n} \in \partial B\left(x_{0}, r\right)$. Now the condition (A3) applies since $x_{n} \rightarrow x$ (up to subsequence) and $y_{n} \rightarrow y_{0}$. We then obtain $x_{n} \rightarrow x \in \partial B\left(x_{0}, r\right), x \in D(T)$ and $y_{0} \in T(x)$. This contradicts $y_{0} \notin T\left(D(T) \cap \partial B\left(x_{0}, r\right)\right)$.

Since $B\left(y_{0}, \delta\right) \cap T\left(D(T) \cap \partial B\left(x_{0}, r\right)\right)=\emptyset, y_{0} \in T\left(D(T) \cap B\left(x_{0}, r\right)\right)$ and the ball $B\left(y_{0}, \delta\right)$ is pathwise connected, we can apply Proposition 2.1 to obtain $B\left(y_{0}, \delta\right) \subset T\left(D(T) \cap B\left(x_{0}, r\right)\right)$.

It would be interesting to establish analogous results via degree theory for operators of the form $A+T$, where $A: X \supset D(A) \rightarrow 2^{X^{*}}$ is maximal monotone and $T$ is in $\mathcal{A}_{G}\left(S_{+}\right)$. Similar results are expected for the sum $L+A+T$ in the spirit of results in [1], where $L$ is densely defined linear maximal monotone operator and $T$ in class $A_{G}\left(S_{+}\right)$with respect to $D(L)$.

Acknowledgment: This research is partially supported by the College of Science and Mathematics at Kennesaw State University through the 2016 Research Stimulus Program.

\section{REFERENCES}

[1] D.R. Adhikari and A.G. Kartsatos, Invariance of domain and eigenvalues for perturbations of densely defined linear maximal monotone operators, Applicable Analysis 95 (2016), no. 1, 24-43.

[2] J. Berkovits, On the degree theory for nonlinear mappings of monotone type, Ann. Acad. Sci. Fenn. Ser. A I, Math. Dissertationes 58 (1986).

[3] L.E.J. Brouwer, Über Abbildungen von Mannigfaltigkeiten, Math. Ann. 71 (1912), 97-115.

[4] K. Deimling, Zeros of accretive operators, Manuscr. Math. 13 (1974),), 365-374.

[5] A.G. Kartsatos, Zeros of demicontinuous accretive operators in reflexive banach spaces, J. Integral Equations 8 (1985), 175-184.

[6] Sets in the ranges of nonlinear accretive operators in banach spaces, Studia Math. 114 (1995), 261-273.

[7] A.G. Kartsatos and I.V. Skrypnik, Degree theories and invariance of domain for perturbed maximal monotone operators in Banach spaces, Adv. Differential Equations 12 (2007), 1275-1320.

[8] A. Kittilä, On the topological degree for a class of mappings of monotone type and applications to strongly nonlinear elliptic problems, Ann. Acad. Sci. Fenn. Ser. A I Math. Dissertations 91 (1994), 48pp.

[9] M. Nagumo, Degree of mapping in convex linear topological spaces, Amer. J. Math. 73 (1951), 497-511. 
[10] J.A. Park, Invariance of domain theorem for demicontinuous mappings of type $\left(S_{+}\right)$, Bull. Korean Math. Soc. 29 (1992), no. 1, 81-87.

[11] W. V. Petryshyn, Invariance of domain theorem for locally A-proper mappings and its implications, J. Funct. Anal. 5 (1970), 137-159.

[12] _ Approximation-solvability of nonlinear functional and differential equations, Marcel Dekker, New York, 1993.

[13] J. Schauder, Invarianz des gebietes in funktional raümen, Studia Math. 1 (1929), 123-130.

[14] I.V. Skrypnik, Methods for analysis of nonlinear elliptic boundary value problems, Ser. II, vol. 139, Amer. Math. Soc. Transl., Providence, Rhode Island, 1994.

[15] S.L. Trojanski, On locally uniformly convex and differentiable norms in certain non-separable Banach spaces, Studia Math. 37 (1971), 173-180.

[16] A.J. Tromba, Some theorems on fredholm maps, Proc. Amer. Math. Soc. 34 (1972), 578-585. 